\title{
Política agropecuaria y pobreza rural: reflexiones a partir de dos casos en la Región Chorotega, Costa Rica
}

\section{Agricultural Policy and Rural Poverty: Reflections from Two Cases in the Chorotega Region, Costa Rica}

\author{
Sandra Lezcano Calderón \\ Universidad Nacional a \\ Sede Regional Chorotega \\ Guanacaste, Costa Rica \\ Sandra.lezcano.calderon@una.cr \\ Silvia Zúñiga Guerrero \\ Universidad Nacional \\ Sede Regional Chorotega \\ Guanacaste, Costa Rica \\ Silvialo2007@yahoo.es \\ María Teresa Dobles Villegas \\ Universidad Nacional \\ Recinto Sarapiquí \\ Heredia, Costa Rica \\ maria.dobles.villegas@una.cr
}

Recibido 02/06/2017 Aceptado 06/09/2017

Resumen. El artículo es una revisión de las políticas del sector agropecuario, a partir de los resultados de un proyecto de extensión con pequeños productores, y uno de investigación, referido a articulaciones interinstitucionales, desarrolladas en la Región Chorotega, en Costa Rica. Este trabajo tiene como objetivo evidenciar los vacíos existentes en las políticas del sector agropecuario costarricense, relacionadas con la reducción de la pobreza y generación de empleo, dirigido a los pequeños productores agropecuarios.

Palabras clave: población rural, políticas agropecuarias, pobreza, productor agrícola. 
Revista Universidad en Diálogo • Vol. 7, N. 2, Julio-Diciembre, 2017, pp. 73-96

ISSN 2215-2849 • EISSN: 2215-4752

DOI: http://dx.doi.org/10.15359/udre.7-2.4

\begin{abstract}
The article reviews the policies of the agricultural sector. The review is based on the results of two projects: one was an extension project implemented with small producers; and the other one was a research project related to inter-institutional articulations, conducted in the Chorotega Region in Costa Rica. This paper aims to highlight the gaps in Costa Rican agricultural sector policies related to the reduction of poverty, and the generation of employment directed to small agricultural producers.
\end{abstract}

Keywords: agricultural policies, rural population, poverty, agricultural producer.

\title{
Introducción
}

La pobreza es un tema complejo, multifacético y heterogéneo, difícil de definir, explicar y medir satisfactoriamente. Es una condición en la cual, una o más personas, tienen un nivel de bienestar inferior al mínimo necesario para la sobrevivencia, y presentan limitaciones para superarla, pues se requiere de un sistema integrado de desarrollo social, para que las personas salgan de esa condición.

En Costa Rica, algunas políticas de Estado, están dirigidas a disminuir la pobreza, la desigualdad y aumentar las posibilidades de empleo. Estas políticas establecen su población objetivo, a partir de una caracterización cuantitativa, basada en la estimación de ingresos y la propiedad privada máxima.

En la definición de las políticas, no se incluye, en forma explícita, la caracterización o competencias básicas que deben cumplir sus destinatarios, tales como: nivel de escolaridad, experiencia en el sector productivo o laboral. Estas competencias, en la práctica, son primordiales para poder superar los trámites, de lo que depende el cumplimiento de las políticas públicas, convirtiéndose en un filtro que impide el adecuado acceso a ellas.

En Costa Rica, se utiliza la medición de ingresos para valorar la incidencia de la pobreza, a pesar de que se reconoce su insuficiencia metodológica para la medición de ella. Según este criterio, la Encuesta Nacional de Hogares (ENAHO) 2014, arrojó que el 22,4 \% de la población total del país, se encuentra en esta condición, pero con marcadas diferencias urbano-rural (el $30,3 \%$ rural y el $19,5 \%$ urbana).

En la estrategia oficial, se establece los mecanismos operativos para lograr la disminución de la pobreza, en función de las competencias institucionales, de tal manera, se propone que los sujetos de estas políticas, requieren solicitarlo, únicamente, para beneficiarse de ellas. 
Desde dicho planteamiento, se asume que las instituciones responsables de ejecutar las políticas tienen conocimiento completo de las características de esta población (ubicación, experiencia, nivel de comprensión de los procedimientos requeridos para acceder a los recursos), así como la capacidad de convocatoria y canales de comunicación adecuados con la población objetivo.

En lo referido al sector agropecuario, se busca que este sea más competitivo, y se desprende que esa competitividad coincide con el concepto de competitividad auténtica, que "no es simplemente un proceso continuo de inversión, sino que debe estar ligada también a un aumento sostenido de los niveles de vida de la población" (Suñol, 2006, p. 183).

El autor precitado, señala que en economías de escaso desarrollo, es necesario trabajar sobre lo que se denomina determinantes internos: la educación, destrezas para el uso adecuado de los equipos, mejoras en la infraestructura, eficiencia ecológica, tecnología e innovación.

El factor humano es fundamental para mejorar la competencia, ya que aumenta la productividad, pues esto generará riqueza y crecimiento económico, que beneficiará a la población con un mejor nivel de vida, y se disminuirá la pobreza (Marín-Raventós, 2012 y Tam, 2016).

El presente análisis, se realiza a partir de los resultados de dos proyectos desarrollados en la Región Chorotega, en Costa Rica. Uno de extensión universitaria denominado "Fortalecimiento de capacidades locales para la gestión de proyectos en organizaciones sociales del sector agropecuario en Guanacaste" (FCL), que acompañó a dos asociaciones de pequeños productores agrícolas en la formulación de un proyecto, con el propósito que el sector agropecuario se los financiara.

Asimismo, otro de investigación intitulado "Fomento de las articulaciones para el desarrollo humano local en la Región Chorotega, Costa Rica" (FA), que identificó actores, espacios de articulación y proyectos en la Región Chorotega.

Este trabajo tiene como objetivo: evidenciar los vacíos existentes en las políticas del sector agropecuario costarricense, relacionadas con la reducción de la pobreza y generación de empleo, dirigido a los pequeños productores agropecuarios. 
ReVista Universidad en Diálogo • Vol. 7, N. ², Julio-Diciembre, 2017, pp. 73-96

ISSN 2215-2849 • EISSN: 2215-4752

DOI: http://dx.doi.org/10.15359/udre.7-2.4

\section{Metodología}

El trabajo se desarrolló desde el enfoque cualitativo, y es de carácter descriptivo. Se revisó la Política de Estado para el Sector Agroalimentario y el Desarrollo Rural Costarricense 2010-2021 (PESA), el Plan Nacional de Desarrollo 2014-2018 (PND) y el Plan Sectorial de Desarrollo Agropecuario y Rural 2015-2018 (PSDAR), donde plantean estos instrumentos, con respecto a la caracterización de los pequeños productores, así como los objetivos, las metas e indicadores relacionados.

Los resultados de la revisión documental fueron comparados con lo encontrado en los dos proyectos desarrollados en la Región Chorotega.

Esto para responder a las preguntas ¿Por qué los pequeños agricultores se mantienen en la pobreza? ¿Por qué organizaciones de pequeños agricultores que son sujeto de los servicios del sector agropecuario, no pueden insertarse en el mercado local de manera competitiva y, con ello, incrementar sus ingresos y generar empleo?

\section{Contextualización de las experiencias}

La formulación del proyecto FCL responde a un esfuerzo de articulación interinstitucional entre el Ministerio de Agricultura y Ganadería (MAG) en la región Chorotega y la Sede Regional Chorotega de la Universidad Nacional de Costa Rica, que tuvo como propósito, contribuir con el desarrollo de capacidades en las organizaciones de pequeños productores agropecuarios, para que se vinculen con el Mercado Regional Mayorista de la Región Chorotega, un proyecto que pretende brindar "a unos 17500 productores una infraestructura y un canal directo para ofrecer sus productos" (Bermúdez, 2015), que inició su construcción durante el 2017.

El proyecto FCL tenía como propósito desarrollar capacidades locales mediante un proceso de acompañamiento a dos organizaciones de pequeños productores agrícolas en la formulación de proyectos. Inicialmente, las organizaciones tenían definido presentar un proyecto para que les financiaran la infraestructura de un centro de acopio en cada una de sus comunidades, ya que consideraban que, con esto, se resolvería su problemática de comercialización de sus productos - lo que implicaba ingresos y empleo para las familias-, y podrían vincularse con el Mercado Regional Mayorista de la Región Chorotega, cuando entrara en funcionamiento. 
En el proceso de capacitación, por parte del proyecto FCL, ambas organizaciones determinaron que, para generar mayores ingresos, crear opciones de empleo y mejores condiciones de trabajo en la comunidad, no era suficiente contar con centros de acopio, sino mejorar las condiciones de producción, la calidad del producto, conocer los canales de comercialización de los productos agrícolas, e insertarse en el mercado de forma permanente.

Lo anterior, llevó a cada organización a formular un proyecto que les permitiera lograr lo siguiente:

- Un estudio de mercado para conocer la demanda de productos agrícolas y los estándares de calidad que requiere el mercado local y, con ello, orientar la producción de sus asociados.

- Una estrategia de comercialización que integre a sus asociados, pues cada agricultor produce y vende individualmente, condición que nos les permite ofrecer volumen, variedad ni calidad estandarizada para acceder a mercados formales.

- Una producción planificada de los cultivos agrícolas de los afiliados, para contar con una oferta constante, según la demanda.

- Conocimiento del potencial de las unidades productivas, para identificar alternativas de diversificación o mejora de los cultivos.

- Proceso de capacitación, orientado al mejoramiento de las técnicas de producción y estandarización de la calidad para su comercialización.

- Desarrollo de las capacidades gerenciales para la ejecución de un proyecto y el desarrollo de sentido de pertinencia de los asociados con la organización (Asociación de Productores Agropecuarios de Río Naranjo, 2016 y Asociación de Productores Agropecuarios de Cañas Dulces, 2016).

A todo este pliego de peticiones, consideradas indispensables para la inserción permanente de ambas organizaciones en el mercado, la respuesta de las instituciones vinculadas con el sector agropecuario y social, fue que no podían brindar dichos servicios, ya que había recursos, únicamente para la inversión.

Esto hace referencia a que solo se podía financiar transferencia tecnológica, que implicara productos tangibles, tales como equipos, insumos o infraestructura, 
Revista Universidad en Diálogo • Vol. 7, N. 2, Julio-Diciembre, 2017, pp. 73-96

ISSN 2215-2849 • EISSN: 2215-4752

DOI: http://dx.doi.org/10.15359/udre.7-2.4

pero que no eran posibles procesos formativos y de acompañamiento para la mejora de las competencias básicas de los agricultores para acceder al mercado.

Sin embargo, en la investigación del proyecto FA, como indican Zúñiga y Lezcano, 2015, en donde se identificó actores, espacios de articulación y proyectos en la Región Chorotega, se determinó que existe una oferta amplia de servicios que cubre todo el proceso de producción, orientados a incrementar la competitividad y mejores empleos, donde la mayoría de los proyectos o actividades que se encuentra, se ubica en las categorías denominadas mercados y comercialización, desarrollo de capacidades asociadas con la producción y asistencia técnica para el mejoramiento de procesos y, con menor frecuencia, es la de gestión empresarial.

Lo anterior indica que, si bien es cierto existen proyectos y acciones que se realizan en la región, orientadas a incrementar la competitividad, estas no tienen las características requeridas por los pequeños productores agrícolas en estudio, que les permita mejorar sus capacidades y poder vincularse al mercado (Zúñiga y Lezcano, 2015).

Según Zúñiga y Lezcano (2015), por normativa, existen dos espacios de coordinación interinstitucional vinculados directamente con el sector agropecuario, a saber: el Comité Sectorial Regional Agropecuario (CSRA), coordinado por el MAG, cuyo alcance es provincial, y del cual derivan los Comités Sectoriales Locales (COSELES), que funcionan en los cantones y el Foro Nacional Mixto, constituido por representantes de los foros regionales mixtos.

Los COSELES tienen como mandato, realizar sus funciones sectoriales de forma armónica e integrada, que contribuyan con el desarrollo regional, mejorar el rendimiento y efectividad de los servicios de las instituciones públicas en la región, vincular los proyectos del sector agropecuario con los sectores relacionados, especialmente, con recursos naturales, salud, educación y gobiernos locales; asimismo, mantener relación directa con las organizaciones de productores, a fin de identificar sus necesidades y buscar soluciones conjuntas a su problemática (Decreto 32488-MAG, 2005,29 de julio).

El Foro Regional Mixto constituido por el MAG y formado por representantes de diferentes organizaciones de productores agropecuarios de la región, con un representante en el Foro Mixto Nacional, tiene como propósito informar, analizar y proponer acciones para el desarrollo del sector agroalimentario, las economías de los territorios rurales, fortalecer el crecimiento 
de la organización social en la región, que permita aumentar los niveles de competitividad e impulsar programas y proyectos de desarrollo, que aseguren el crecimiento económico y social de los territorios rurales. Así mismo, todos aquellos asuntos del espacio regional del sector agroalimentario, que no sean resueltos en dicho ámbito, formarán parte de la agenda del Foro Regional Mixto (Decreto N 36828-MAG, 2011, 2 noviembre).

Además, los Consejos Cantonales de Coordinación Interinstitucional (CCCI), coordinados por el gobierno local, que elabora un plan anual, orientado a satisfacer las necesidades reales de cada cantón, en el cual se debe conciliar los programas y los presupuestos de los entes públicos, para una mayor eficacia y eficiencia en la acción pública, que incluye recomendar los cambios necesarios, con el fin de ajustarlos a las necesidades locales, ante lo cual, las instituciones tomarán las medidas necesarias, para incorporarlas como parte de su plan anual operativo.

Dichos planes serán evaluados para garantizar la ejecución de los objetivos y las metas propuestas para cada año. Además, tiene la potestad de identificar debilidades en las políticas públicas de naturaleza local, con el propósito de proveer la acción subsidiaria de otras instancias de naturaleza nacional, en atención del interés público local (Decreto Ejecutivo No 36004-PLAN (13 de mayo del 2010).

En resumen, según los resultados del proyecto de F.A., si bien es cierto que en la región, la oferta no responde a las necesidades del pequeño productor agrícola, con características semejantes a los casos en estudio, también es cierto, que existen espacios de articulación interinstitucional, que tienen las competencias para modificarla.

Sin embargo, la situación se convierte en un círculo vicioso, porque para que el pequeño productor acceda a los recursos institucionales disponibles, debe desarrollar las capacidades y el desarrollo de estas capacidades no forma parte de la oferta institucional.

\section{Caracterización de un pequeño y mediano agricultor desde la política pública}

En el país, según Secretaría Ejecutiva de Planificación Sectorial Agropecuaria [SEPSA] (2015), el sector agropecuario identifica tres tipos de productores, a saber: 
Revista Universidad en Diálogo • Vol. 7, N. 2, Julio-Diciembre, 2017, pp. 73-96

ISSN 2215-2849 • EISSN: 2215-4752

DOI: http://dx.doi.org/10.15359/udre.7-2.4

- Los pequeños productores, quienes producen para autoconsumo y venden sus excedentes en el mercado interno.

- Los pequeños y medianos productores que abastecen el mercado interno y exportan pequeños volúmenes.

- Pequeños, medianos y grandes productores con mayor nivel empresarial y de tecnificación, cuyos productos son para la exportación, y solo los excedentes o productos que no califican para la exportación se colocan en el mercado nacional.

En las áreas rurales del país, existen rezagos importantes en su desarrollo, tal y como se observa en la tabla 1 , lo cual evidencia las posibilidades del pequeño productor de incorporarse, en forma permanente, en los procesos de desarrollo económico y social.

Tabla 1

Distribución de las personas en Costa Rica porzona, según condición de pobreza multidimensional Julio 2015 y 2016

\begin{tabular}{|c|c|c|c|c|c|c|c|}
\hline \multirow[t]{3}{*}{ Año } & \multirow[t]{3}{*}{ Zona } & \multirow{2}{*}{\multicolumn{2}{|c|}{ Total }} & \multicolumn{4}{|c|}{ Pobreza multidimensional } \\
\hline & & & & \multicolumn{2}{|c|}{ No pobres } & \multicolumn{2}{|c|}{ Pobres } \\
\hline & & Absoluto & Relativo & Absoluto & Relativo & Absoluto & Relativo \\
\hline \multirow[t]{3}{*}{2015} & $\begin{array}{l}\text { Total de } \\
\text { personas }\end{array}$ & 4823715 & 100,0 & 3561496 & 73,8 & 1262219 & 26,2 \\
\hline & Urbano & 3503467 & 100,0 & 2780880 & 79,4 & 722587 & 20,6 \\
\hline & Rural & 1320248 & 100,0 & 780616 & 59,1 & 539632 & 40,9 \\
\hline \multirow[t]{3}{*}{2016} & $\begin{array}{l}\text { Total de } \\
\text { personas }\end{array}$ & 4878135 & 100,0 & 3656666 & 75,0 & 1221469 & 25,0 \\
\hline & Urbano & 3542355 & 100,0 & 2847828 & 80,4 & 694527 & 19,6 \\
\hline & Rural & 1335780 & 100,0 & 808838 & 60,6 & 526942 & 39,4 \\
\hline
\end{tabular}

Nota. INEC. Encuesta Nacional de Hogares (ENAHO), 2015 y 2016. 
En estas áreas rurales, los pequeños productores requieren de:

- Mayor acceso a tecnologías de producción.

- Regulación y disponibilidad de material genético y semilla de calidad.

- Implementación de medidas fito, zoosanitarias y de reglamentación técnica.

- Mayor agregación de valor e integración de la producción a los circuitos comerciales locales y regionales.

- Fortalecer la capacidad gerencial de sus organizaciones de productores y productoras.

- Fortalecer los niveles de organización.

- Disponibilidad de financiamiento adecuado a las necesidades de las diferentes actividades productivas.

- Mejorar la calidad e inocuidad de los productos.

- Servicios básicos de Maquinaria y equipo.

- Disponibilidad de tierra.

Adicionalmente, estos agricultores tienen bajos niveles de escolaridad, en donde el 86,18 \% no ha completado la secundaria (el 5,3\% no lee ni escribe; el 28,4 \% tiene primaria incompleta; el $42,6 \%$ primaria completa, y el 9,8\% secundaria incompleta), según se desprende del último Censo Nacional Agropecuario, realizado en el 2014, tal y como se muestra en la tabla 2. Con respecto a los datos recuperados de los cantones Liberia y Bagaces, en comparación con los totales de Guanacaste y Costa Rica.

La situación anterior, coincide con lo encontrado en las dos organizaciones de productores agrícolas analizadas.

A continuación, se detalla algunas de sus características con respecto a la educación formal recibida por las personas participantes en el proyecto FCL.

Si se toma en consideración los porcentajes de personas que no saben leer ni escribir, más las personas que tienen primaria incompleta y primaria completa, se tiene que el 58,06\% de quienes participaron en el proceso no asistió a la secundaria, y un porcentaje importante no alcanzó siquiera concluir la primaria (el 29,03\%). 
Revista Universidad en Diálogo • Vol. 7, N. 2, Julio-Diciembre, 2017, pp. 73-96 ISSN 2215-2849 • EISSN: 2215-4752

DOI: http://dx.doi.org/10.15359/udre.7-2.4

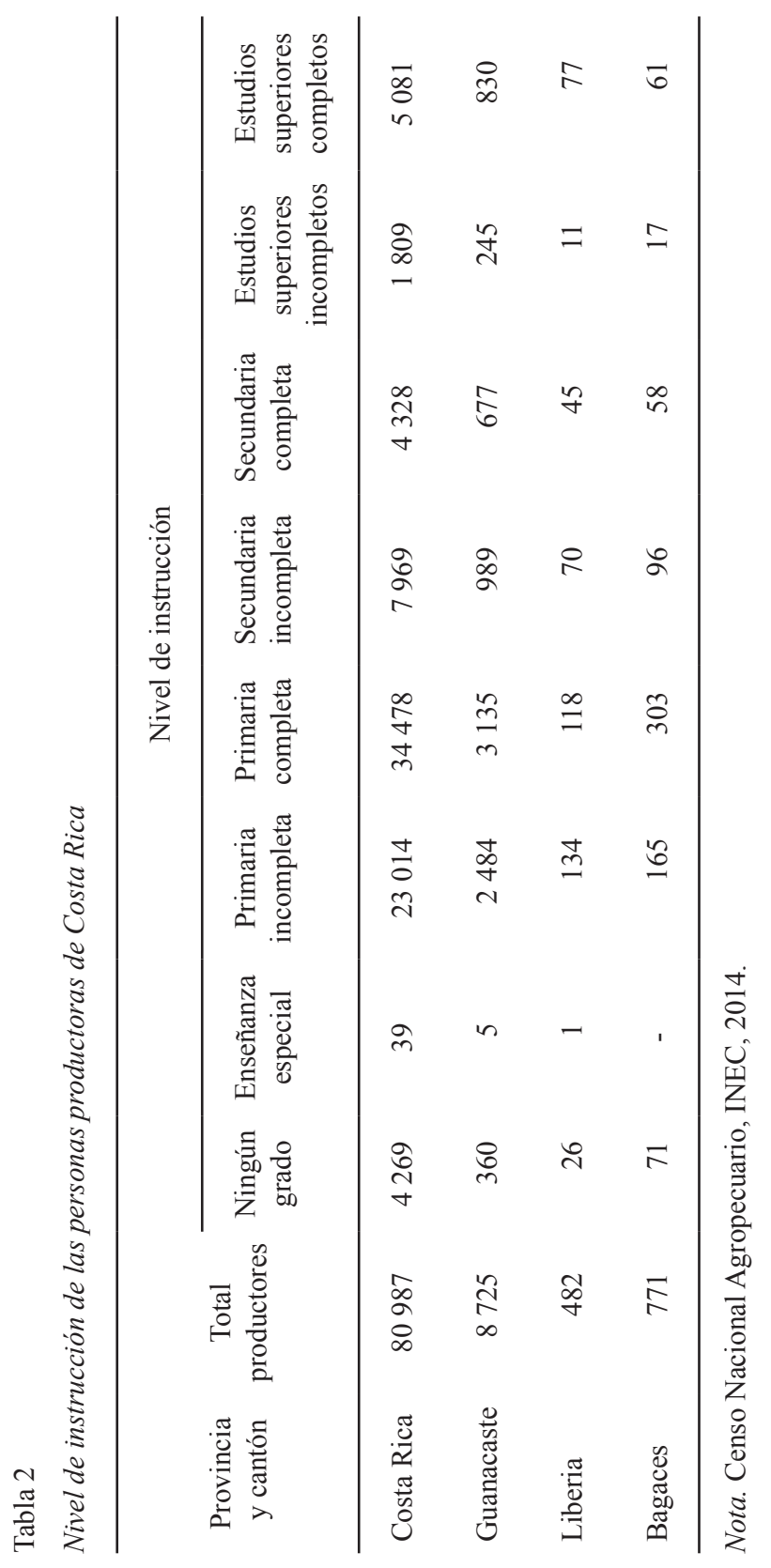


Tabla 3

Nivel de instrucción de los afiliados de las dos asociaciones de pequeños agricultores participantes en el proyecto FCL

\begin{tabular}{lccccc}
\hline $\begin{array}{c}\text { Escolaridad de los } \\
\text { participantes en el } \\
\text { proyecto }\end{array}$ & $\begin{array}{c}\text { Asociación } \\
\text { de Río } \\
\text { Naranjo }\end{array}$ & $\begin{array}{c}\text { Asociación } \\
\text { de Cañas } \\
\text { Dulces }\end{array}$ & Total & $\begin{array}{c}\text { Total } \\
(\%)\end{array}$ & $\begin{array}{c}\text { Total } \\
\text { acumulado } \\
(\%)\end{array}$ \\
\hline No leen ni escriben. & 1 & 1 & 2 & 6,45 & 6,45 \\
Primaria incompleta & 3 & 2 & 5 & 16,13 & 22,58 \\
Primaria completa & 8 & 3 & 11 & 35,48 & 58,06 \\
Secundaria incompleta & 2 & 1 & 3 & 9,68 & 67,74 \\
Secundaria completa & 3 & 3 & 6 & 19,36 & 87,1 \\
Universitaria incompleta & 0 & 2 & 2 & 6,45 & 93,55 \\
Universitaria completa & 0 & 2 & 2 & 6,45 & 100 \\
\hline Total & 17 & 14 & 31 & 100 & \\
\hline
\end{tabular}

Nota. Elaboración propia, a partir de información del Proyecto de FCL: Diagnóstico de necesidades de capacitación en TIC's.

Lo anterior coincide con los datos de la Encuesta Nacional de Hogares (ENAHO), realizado por el Instituto Nacional de Estadística y Censos de Costa Rica (INEC), en el año 2016, del que se desprende que el 56,78 \% de la población rural de Costa Rica no pasa a secundaria (el 6,67 \% sin instrucción; el 18, 22 \% primaria incompleta y el 31,89 \% primaria incompleta).

En lo que se refiere a la secundaria, el 32,26\% (10) de los 31 participantes en el proyecto FCL la completaron; incluye a quienes ingresaron en la universidad, sea que hayan completado o no la educación superior, porcentaje mayor al 21,39\%, correspondiente al promedio nacional de la población rural del país, según la ENAHO, 2016 (INEC, 2016). 
Considerando los datos anteriores, se puede inferir que el 58,06 \% de los participantes del proyecto no tuvieron la formación académica que les permitiera desarrollar las competencias de análisis y síntesis de información, aspectos que son esenciales en el desarrollo y formulación de un proyecto y una planificación estratégica de la organización social, elementos que van de la mano, y que no solo son requerimientos para ser beneficiarios de apoyo institucional, sino que también aumenta las posibilidades de éxito en la ejecución de los proyectos formulados.

\section{Tabla 4}

Utilización de TICS por los afiliados de las dos asociaciones de pequeños agricultores participantes en el proyecto $F C L$

\begin{tabular}{lccc}
\hline $\begin{array}{c}\text { Utilización de TICs por } \\
\text { los participantes en el } \\
\text { proyecto }\end{array}$ & $\begin{array}{c}\text { Asociación de } \\
\text { Río Naranjo } \\
\text { (17 participantes) }\end{array}$ & $\begin{array}{c}\text { Asociación de } \\
\text { Cañas Dulces } \\
\text { (14 participantes) }\end{array}$ & $\begin{array}{c}\text { Total } \\
\text { Participantes } \\
\text { utilizan TICs }\end{array}$ \\
\hline $\begin{array}{l}\text { Pueden utilizar una } \\
\text { computadora }\end{array}$ & 1 & 3 & 4 \\
$\begin{array}{l}\text { Manejan paquete office } \\
\text { o similares para digitar } \\
\text { información }\end{array}$ & 1 & 3 & 4 \\
$\begin{array}{l}\text { Pueden utilizar Internet } \\
\text { Total }\end{array}$ & 8 & & 14 \\
\hline
\end{tabular}

Nota. Elaboración propia, a partir de información del Proyecto de FCL: Diagnóstico de necesidades de capacitación en TIC's.

Como se puede deducir de la tabla 4, el 81,10\% de los participantes no tenía competencias para la mecanografía y procesamiento de información por medio de dispositivos tecnológicos, competencia necesaria para cumplir con el requisito básico de redactar un perfil de proyecto, para solicitar financiamiento o apoyo a una institución.

Además, el uso de Internet por el 45,16 \% de los participantes, en realidad, hace referencia al uso ocioso de aplicaciones relacionadas con el entretenimiento y la comunicación inmediata a través de teléfonos celulares, desde donde acceden a redes sociales, por lo que este indicador no refleja la potencialidad de desarrollar investigaciones y consultas en la web. 
Por lo anterior, considerando las estadísticas generadas por el INEC y los casos en estudio, se cuestiona si los requerimientos y los mecanismos de acceso al cumplimiento de las políticas públicas están adaptados, realmente, a las personas del sector que más apoyo necesitan por sus índices de pobreza.

Lo anterior implica analizar, también, las posibilidades reales de los técnicos de campo de las instituciones públicas, en asuntos de promoción de proyectos con un enfoque de desarrollo local, si tomamos en cuenta que, en muchos casos, éstos son recursos únicos en las agencias locales de las instituciones, y los mecanismos de cumplimiento de las políticas son ajenos a la realidad que enfrentan ellos.

En resumen, se identifica una necesidad de potenciar las capacidades que se espera tengan los pequeños agricultores, para que puedan insertarse en el mercado, y esto debe ser desarrollado desde los aspectos básicos de la educación formal, así como la necesidad de acercar los mecanismos de cumplimiento de las políticas públicas a los pequeños productores.

\section{Política pública del Sector agropecuario y su relación con las deman- das de servicios de pequeños agricultores de los casos en estudio}

El PESA 2010-2021 plantea que es necesaria la búsqueda permanente de condiciones que aumenten la competitividad, sostenibilidad, estimule las potencialidades de sus territorios y que haya una mayor equidad e inclusión social (SEPSA, 2011).

Se propone como medios para lograr estos resultados:

- Desarrollar capacidades gerenciales y de toma de decisiones en los productores para la innovación ante el cambio climático.

- Recuperar el potencial productivo.

- Identificar nuevas opciones de producción.

- Mejoramiento de material genético.

- Nuevas prácticas de cultivo, entre otras ([SEPSA], 2011).

Por su parte, el Plan Nacional de Desarrollo (PND), que recoge lo dispuesto en el PESA, plantea que los instrumentos para lograr la competitividad son: el fortalecimiento de los programas de capacitación a productores, empleados o equipos gerenciales de empresas agroindustriales; lo que requiere el 
Revista Universidad en Diálogo • Vol. 7, N. 2, Julio-Diciembre, 2017, pp. 73-96

ISSN 2215-2849 • EISSN: 2215-4752

DOI: http://dx.doi.org/10.15359/udre.7-2.4

diseño de programas de gestión empresarial, mercadeo, logística, manipulación e inocuidad de alimento, uso de tecnologías de información y comunicación, y fomento de organizaciones, entre otras materias (SEPSA, 2011).

En resumen, se pretende que las organizaciones de productores cuenten con información, mejores tecnologías de información y comunicación para el acceso oportuno de dónde, cómo, cuándo y en qué condiciones vender, de manera que estén preparados para que puedan articularse con éxito a los mercados (Ministerio de Planificación Nacional y Política Económica [MIDEPLAN], 2014).

Así las condiciones, la solicitud de las asociaciones de agricultores en estudio no difiere con lo establecido en el PESA y el PND. Entonces, ¿Por qué no fue posible brindarles los servicios solicitados, por parte de las instituciones de la región?

\section{Vacíos de las políticas del sector agropecuario para la reducción de la pobreza}

El sector agropecuario tiene como objetivo estratégico, mejorar la competitividad y el desarrollo rural sostenible y, además, contribuye a cumplir con el objetivo de reducción de la pobreza, siendo este último un mandato subsidiario.

Las instituciones encargadas de reducir los índices de pobreza son el Ministerio de Trabajo y Seguridad Social (MTSS), Ministerio de Educación Pública (MEP), Ministerio de Salud (MS), y el Ministerio de Vivienda y Asentamientos Humanos (MIVAH).

Dichas instituciones ejecutan políticas de reducción de la pobreza con enfoque asistencial, no con un enfoque de desarrollo de capacidades, que permita resolver la situación de manera estructural, lo que limita las posibilidades de salir de la pobreza por parte de los sectores atendidos.

Sin embargo, las organizaciones de pequeños productores agropecuarios, que se atiende, actualmente, por el sector agropecuario, y se pretende vincular con el mercado, como los casos en estudio, tienen características socioeconómicas que los enmarcan como sujetos en condiciones de pobreza o vulnerabilidad. No obstante, el desarrollo de capacidades no es prioritario en las políticas del Sector Agropecuario, lo que es medular para que se inserten en el sector productivo. 
Entendiendo desarrollo de capacidades como la habilidad de una persona para hacer actos que para ella es valioso alcanzar y que tiene que "ver con problemas sociales que afectan al bienestar humano, tales como la desigualdad, la pobreza, la calidad de vida, la ausencia de desarrollo humano y la injusticia social" (Urquijo, 2014).

Entre esas habilidades se encuentra la de "movimiento, la habilidad de satisfacer ciertas necesidades alimentarias, la capacidad de disponer de medios para vestirse y tener alojamiento, o la capacidad de participar en la vida social de la comunidad (Sen, 1982 citado por Urquijo, 2014, p. 66).

En la tabla 5, se colocó seis de los 37 resultados, indicadores y riesgos planteados para el Sector Agropecuario en el PND 2015, con el propósito de ilustrar sus características. Del total de resultados, solo uno está relacionado con el desarrollo de capacidades, que les permita aprovechar y administrar recursos, el resto están vinculados directamente con el desarrollo de capacidades de producción.

En el resultado referido al desarrollo de capacidades, su énfasis es en el aspecto de carácter técnico de producción, y se dirige, principalmente, a jóvenes rurales.

Por otro lado, los riesgos se plantean, en función de las limitaciones institucionales. La mayoría de ellas, no están asociadas con la institución responsable del producto, sino a otras que deberían acompañar en el proceso, y tampoco se identifica limitaciones asociadas con el sujeto, al cual se dirige la política. No se indica quién tiene esa competencia; no se identifica como riesgo y, por tanto, tampoco se gestiona desde la planificación.

Entonces, lo anterior evidencia que toda la información que hay en el diagnóstico realizado está ausente en la aplicación de la política. ¿Esta ausencia de información responde a limitaciones del instrumento que se utiliza o a que, definitivamente, quienes hicieron el PND, no consideraron el diagnóstico?

Independientemente de las razones por las que no se refleja el desarrollo de capacidades en el PND, lo cierto es que las instituciones responden en su rendición de cuentas a las metas e indicadores de gestión establecidos en este plan, por lo tanto, si éstos corresponden únicamente al área de producción, los funcionarios trabajarán para cumplir solo metas de producción programadas. 
Revista Universidad en Diálogo • Vol. 7, N. 2, Julio-Diciembre, 2017, pp. 73-96

ISSN 2215-2849 • EISSN: 2215-4752

DOI: http://dx.doi.org/10.15359/udre.7-2.4

Tabla 5

Oferta de servicios considerada en la estrategia del sector agropecuario contenida en el Plan Nacional de Desarrollo 2014-2018

\begin{tabular}{|c|c|c|}
\hline Resultados & Indicadores & Riesgos \\
\hline $\begin{array}{l}\text { Aumento del valor } \\
\text { agregado agropecuario. }\end{array}$ & $\begin{array}{l}\text { Tasa de crecimiento } \\
\text { del Valor Agregado } \\
\text { Agropecuario. }\end{array}$ & $\begin{array}{l}\text { Sectores complementarios } \\
\text { que deben involucrarse no } \\
\text { lo hagan. }\end{array}$ \\
\hline $\begin{array}{l}\text { Reducción de la pobreza } \\
\text { rural en } 6,5 \text { puntos. }\end{array}$ & $\begin{array}{l}\text { Porcentaje de hogares } \\
\text { rurales en pobreza. }\end{array}$ & $\begin{array}{l}\text { Sectores complementarios } \\
\text { que deben involucrarse no } \\
\text { lo hagan. }\end{array}$ \\
\hline $\begin{array}{l}\text { Desarrollar el conocimiento } \\
\text { tecnológico, la transferencia } \\
\text { de tecnologías ... para } \\
\text { lograr el aumento de } \\
\text { la productividad en los } \\
\text { productos sensibles. }\end{array}$ & $\begin{array}{l}\text { Aumento de rendimiento } \\
\text { por productos sensibles. }\end{array}$ & $\begin{array}{l}\text { Sectores complementarios } \\
\text { que deben involucrarse } \\
\text { no lo hagan, y los factores } \\
\text { climáticos. }\end{array}$ \\
\hline $\begin{array}{l}\text { Aumento de la producción } \\
\text { nacional de café. }\end{array}$ & $\begin{array}{l}\text { Número de hectáreas de } \\
\text { café renovadas, atendidas y } \\
\text { podadas. }\end{array}$ & $\begin{array}{l}\text { Depende de actores } \\
\text { institucionales. }\end{array}$ \\
\hline $\begin{array}{l}\text { Desarrollo y fortalecimiento } \\
\text { agroempresarial de } \\
\text { organizaciones de } \\
\text { productores con equidad } \\
\text { de género, juventud rural, } \\
\text { etnias, que aumentan el } \\
\text { acceso de servicios. }\end{array}$ & $\begin{array}{l}\text { Número de nuevas } \\
\text { organizaciones apoyadas } \\
\text { con capacitación técnica y } \\
\text { empresarial para mejorar } \\
\text { su gestión y el desarrollo } \\
\text { de emprendimientos } \\
\text { agroproductivos sostenibles. }\end{array}$ & $\begin{array}{l}\text { Deficiente gestión operativa } \\
\text { para la adecuada atención } \\
\text { de organizaciones para los } \\
\text { emprendimientos. }\end{array}$ \\
\hline $\begin{array}{l}\text { Productores (as) y jóvenes } \\
\text { rurales desarrollando buenas } \\
\text { prácticas productivas en sus } \\
\text { sistemas productivos, que } \\
\text { posicionen su inserción en } \\
\text { los mercados. }\end{array}$ & $\begin{array}{l}\text { Número de nuevos sistemas } \\
\text { Agroproductivos de } \\
\text { agricultura familiar con } \\
\text { asistencia técnica en } \\
\text { prácticas de producción } \\
\text { Sostenible y orgánica. }\end{array}$ & $\begin{array}{l}\text { Limitado interés y de } \\
\text { recursos de parte de } \\
\text { productores (as) y jóvenes } \\
\text { rurales para la adopción de } \\
\text { metodologías de agricultura } \\
\text { Sostenible y orgánica. }\end{array}$ \\
\hline $\begin{array}{l}\text { Micros, pequeñas y } \\
\text { medianas agroempresas } \\
\text { que comercializan en el } \\
\text { mercado institucional. }\end{array}$ & $\begin{array}{l}\text { Número de nuevas micro, } \\
\text { pequeña y mediana agro } \\
\text { empresas insertadas en } \\
\text { mercado institucional (PAI). }\end{array}$ & $\begin{array}{l}\text { Depende de actores } \\
\text { institucionales. }\end{array}$ \\
\hline
\end{tabular}

Nota. Elaboración propia, a partir de MIDEPLAN, 2015. Se resumió los riesgos establecidos en el PND. 
Por ejemplo, el resultado referido a la contribución a las nuevas tecnologías de riego y drenaje, cuyo indicador es la cantidad de nuevas hectáreas intervenidas con tecnología de riego, se logra con la ampliación del Distrito de Riego Arenal Tempisque, mediante el proyecto de embalse del río Piedras, que tiene proyectado cubrir 149 hectáreas, en esos términos, se alcanza la meta, si se obtiene el préstamo y se adjudica la construcción.

Los vacíos existentes en la política se presentan, debido a que no se contempló las características y condiciones del pequeño agricultor, a pesar de que sí se hace en el diagnóstico, en donde se identifica sus debilidades, sus carencias, y cómo estas provocan la situación en la que se encuentran los pequeños productores.

Se da una concepción adecuada del sujeto, pero en el diseño y la ejecución de la política, no se considera su baja escolaridad, su paquete tecnológico inadecuado para las condiciones de la demanda, la diversidad de paquetes tecnológicos utilizados por los miembros de una misma organización, que genera una oferta colectiva heterogénea $y$, además, la cultura de trabajo individual les demanda un proceso de formación para la generación de una cultura de comercialización colectiva.

Subsanar estos vacíos se ha logrado en algunas agro-cadenas nacionales, como la del café, donde todo el proceso de producción se encuentra estandarizado, todos los agricultores aplican el mismo paquete tecnológico, logran calidad homogénea y la comercialización del producto es conjunto.

Existen aspectos relacionados con el desarrollo de capacidades en las organizaciones, donde no se contempla en la política, y éstos son: la disposición al riesgo y la formación empresarial que les permita proyectar la producción, haciendo una lectura de mercado, sus condiciones actuales y su evolución esperada, es decir, desarrollar capacidad de anticiparse a lo que puede acontecer en el mercado.

\section{Vacíos metodológicos de las políticas del Sector Agropecuario para la reducción de la pobreza}

Existe una desvinculación entre lo que se concibe como características del productor, y lo que se considera en el diseño y ejecución de la política, particularmente, en lo que se refiere a la propuesta para mitigar las debilidades del agricultor, las cuales les impide ser competitivos, debido a: 
Revista Universidad en Diálogo • Vol. 7, N. 2, Julio-Diciembre, 2017, pp. 73-96

ISSN 2215-2849 • EISSN: 2215-4752

DOI: http://dx.doi.org/10.15359/udre.7-2.4

- No se considera los vacíos identificados en el diagnóstico en términos de la necesidad de desarrollar capacidad en las organizaciones para el aprovechamiento de los recursos.

- No se identifica acciones de coordinación del sector agropecuario con las instituciones que tengan esas competencias del desarrollo de capacidades y, que no actúan prioritariamente dentro del sector agropecuario. Es posible que no sea competencia del sector agropecuario el desarrollo de capacidades, pero tampoco aparece ningún resultado o indicador que evidencie que se hará esfuerzos de coordinación de este sector con otras instituciones que sí las tienen, y sobre las cuales, las instituciones deban dar cuentas.

La existencia de esta coordinación interinstitucional (normada y sujeta a sanción por incumplimiento), permitiría que se disminuya en las organizaciones las debilidades mencionadas, y así lleguen al sector agropecuario, con las características requeridas, para poder ejecutar sus acciones vinculadas con la competitividad.

En resumen, que los productores y organizaciones sean capaces de identificar los recursos existentes, direccionarlos y aprovecharlos de la manera que se logre generar ingresos y empleo en las zonas rurales con actividades agropecuarias.

Las acciones identificadas para subsanar los vacíos relacionados con las bajas capacidades de los agricultores, están planteadas para que se desarrolle con estudiantes de los colegios técnicos profesionales, universidades y el INA.

Se tiene, entonces, que al fortalecerse la educación secundaria, para subsanar los vacíos identificados, se resuelve las debilidades de los agricultores que hoy son adultos, quienes están en el campo y no van a insertarse en un proceso formal de educación.

Por lo tanto, se quiere resolver el problema de un sujeto fortaleciendo a otro sujeto distinto al que se dirige la política pública del sector agropecuario, y que, además, si viene de familias de agricultores, tiende a desvincularse de la actividad productiva (desarraigo).

Según indica Dobles (2016) "El deterioro de la imagen de la producción agropecuaria ha incidido en que los jóvenes estudien como una alternativa de proyecto de vida diferente de la de sus padres y no como una posibilidad de mejorar las condiciones y la realidad agropecuaria" (p.135). 
Aunado a lo anterior, el componente de educación, ubicado en el PND, no tiene planteado dentro de sus acciones, actividades dirigidas al Sector Agropecuario, ya que todas son en función de los estudiantes que asisten a la formación, desde sétimo hasta undécimo de enseñanza (III y IV ciclos de educación formal).

Esto evidencia una visión sesgada y dividida del desarrollo local, donde no se considera esfuerzos por aumentar las capacidades educativas básicas en las organizaciones sociales del sector agropecuario. ¿Requiere de mucha inversión a largo plazo y limita las posibilidades de demostrar resultados en el corto plazo?, o ¿Se asume que las capacidades ya existen en los pequeños productores y que el sistema educativo formal las está logrando?

Es decir, que en la ejecución de las políticas dirigidas al sector agropecuario, se visualiza la inversión como el mecanismo para que el pequeño y mediano agricultor sea más competitivo $\mathrm{y}$, con esto, incremente los ingresos y empleos, pues esto disminuirá la pobreza en este sector y, en general, en el espacio rural.

Lo anterior evidencia que existe un vacío metodológico, porque el instrumento no refleja los requerimientos para aumentar las capacidades existentes que tienen los agricultores identificados en el diagnóstico, y validados en las experiencias analizadas, esto hace que las instituciones que ejecutan la estrategia, se pierdan en el desarrollo de las políticas y en la implementación de sus planes operativos institucionales (POI).

Valga decir, que al no estar evidenciado en el PND, las instituciones no lo consideran en la elaboración de sus planes estratégicos, ya que cada una de ellas debe aportar al logro de los objetivos, metas e indicadores de gestión establecidos en el plan.

Si no se rescata en el instrumento, no se va a realizar, porque cada acción que se haga en una institución consume recursos (tiempo de los funcionarios, equipo y recursos tecnológicos), sobre los cuales se debe rendir cuenta.

No se trata de que las instituciones responsables elijan ejecutar o no la política, desarrollar o no capacidades, sino que los instrumentos y la forma en que se planifica no incorporan estos resultados, tampoco identifica acciones e instituciones responsables de hacerlo. 
Revista Universidad en Diálogo • Vol. 7, N. 2, Julio-Diciembre, 2017, pp. 73-96

ISSN 2215-2849 • EISSN: 2215-4752

DOI: http://dx.doi.org/10.15359/udre.7-2.4

Uno de los programas con mayor peso económico ejecutado por esta institución es el de "Transferencia tecnológica", que son recursos de inversión en infraestructura y equipos para este sector, pero no considera dentro de sus rubros el desarrollo de capacidades técnicas de la organización, o los productores, para gestionar las inversiones que se realicen adecuadamente.

Se da esta situación, porque, $¿$ se asume que los agricultores tienen capacidades?, lo cual se contradice con el diagnóstico. ¿Se asume que las instituciones (MEP, universidades, INA) llamadas a desarrollar capacidades lo van a realizar?, porque destinar recursos para desarrollar capacidades en estos sectores, como los casos estudiados, no es una prioridad en el presupuesto nacional.

Probablemente, algunas de las respuestas estén asociadas a que el impacto en el desarrollo de capacidades se evidencia en el mediano plazo y nuestros sistemas de planificación requieren metas visibles en forma anual y de impacto a cuatro años plazo.

Por otro lado, la eficiencia en la administración pública se mide por el porcentaje de ejecución del presupuesto anual asignado, en este sentido, las inversiones en infraestructura y equipamiento alcanzan porcentajes más altos de ejecución del presupuesto en el corto plazo, y los costos de desarrollo de capacidades, aunque son altos en su costo unitario, requieren mayores plazos para ejecutarse, ya que deben acoplarse a la dinámica de la organización; además de ser sistemáticos y constantes.

Una forma de ilustrarlo, es que no se puede tener cinco consultores con una organización de productores para que desarrollen cinco competencias específicas en tres o cinco meses.

Otro elemento para aportar a los vacíos metodológicos en la oferta de servicios institucionales, dirigida a las organizaciones de pequeños productores, es que ella no va dirigida a un mismo sujeto o grupo, y tampoco los servicios que se ofrece son parte de un proceso planificado para lograr las capacidades, porque estas se brindan como acciones puntuales, y no como partes de un proceso global de atención establecido (Zúñiga y Lezcano, 2015). 


\section{Conclusiones}

El PND, como instrumento de planificación, no incluye metas e indicadores relacionados con el desarrollo de las capacidades requeridas por las organizaciones de productores agrícolas pequeños, como en los casos estudiados, a pesar de que en el diagnóstico se identifica la existencia de estas necesidades, las cuales deben subsanarse, si se quiere aumentar la competitividad, sostenibilidad, potencializar los territorios, la equidad, e inclusión social, tal y como indica el PESA 2010-2021.

Unido a lo anterior, la metodología priorizada por el Estado costarricense para implementar las políticas dirigidas al sector agropecuario para la generación de ingresos y empleo, es el desarrollo de proyectos que sean sostenibles económicamente, lo que requiere que los solicitantes manejen el concepto de costos productivos, así como el de utilidad que, la mayoría de las veces, son desconocidas para los pequeños productores.

Finalmente, los formatos utilizados requieren, no solo de capacidades básicas de sistematización, análisis y síntesis de información, sino también del dominio de conceptos y procesos de planificación en la formulación de proyectos, los cuales, muchas veces, no son familiares, ni siquiera para los funcionarios que las promueven.

Se requiere un sistema integrado de desarrollo, si se aspira a que las personas salgan de la situación de pobreza, que sea inclusivo y que defina apoyo desde los procesos de formación de competencias básicas, hasta la formación de competencias empresariales.

En caso contrario, es muy lejano pensar que las políticas públicas contra la pobreza en Costa Rica sean realmente inclusivas, si los medios por los cuáles se pueden hacer efectivas, dejan por fuera a un gran porcentaje de personas y agrupaciones sociales que no cumplen con un ideal formativo, el cual es irreal, en la mayoría de la población rural de nuestro país.

Es importante resaltar que no existen mecanismos para acercar estas políticas a la población, ya que se asume que la política está y que solo debe ser aprovechada, sin analizar las posibilidades reales de beneficio para el grupo meta. 
ReVista Universidad en Diálogo • Vol. 7, N. ², Julio-Diciembre, 2017, pp. 73-96

ISSN 2215-2849 • EISSN: 2215-4752

DOI: http://dx.doi.org/10.15359/udre.7-2.4

\section{Referencias}

Asociación de Productores Agropecuarios de Cañas Dulces. (2016). Proyecto mejoramiento de las capacidades de los productores de Cañas Dulces de Liberia para la producción de calidad y oportunidades de insertarse al mercado. [Documento ubicado en archivos del Drive del Proyecto Fortalecimiento de Capacidades Locales para la gestión de proyectos en organizaciones sociales del sector agropecuario en Guanacaste, Sede Regional Chorotega, Universidad Nacional de Costa Rica].

Asociación de Productores Agropecuarios de Río Naranjo. Proyecto organización de producción y comercialización agrícola de la Asociación de Productores Agropecuarios Río Naranjo. [Documento ubicado en archivos del Drive del Proyecto Fortalecimiento de Capacidades Locales para la gestión de proyectos en organizaciones sociales del sector agropecuario en Guanacaste, Sede Regional Chorotega, Universidad Nacional de Costa Rica].

Bermúdez, M. (2015, 8 de junio). Mercado Regional Mayorista de Región Chorotega beneficiará a 400 mil personas de la zona. Gobiernocr. Recuperado de http://gobierno.cr/mercado-regional-mayorista-deregion-chorotega-beneficiara-a-400-mil-personas-de-la-zona/

Dobles, M.T. (2016). Gestión Comunitaria para el desarrollo local desde la perspectiva de la gestión ambiental con jóvenes y organizaciones base del asentamiento Nueva Guatemala. Tesis para optar por el grado de Magíster Scientiae en Gestión Ambiental y Desarrollo Local. Instituto Centroamericano de Administración Pública.

Decreto $\mathrm{N}^{\circ}$ 36828-MAG. (2011, 2 noviembre). Creación del Foro Nacional Mixto y de los Foros Regionales Mixtos De Organizaciones De Pequeños Y Medianos Productores y del Sector Público Agropecuario. La Gaceta diario oficial del gobierno de la república de Costa Rica, $\mathrm{N}^{\circ} 210$ del 2 de noviembre 2011. Recuperado de http://www.gaceta.go.cr/pub/2011/11/02/COMP_02_11_2011.pdf

Decreto Ejecutivo $\mathrm{N}^{\circ}$ 36004-PLAN. (2010, 13 de mayo). Ley general de transferencia de competencias del poder ejecutivo a las municipalidades. La Gaceta diario oficial del gobierno de la república de Costa Rica, $\mathrm{N}^{\circ} 92$ de 13 de mayo del 2010). Recuperado de https://www. imprentanacional.go.cr/pub/2010/05/04/ALCA7_04_05_2010.pdf 
Decreto 32488-MAG. (2005, 29 de julio). De los Comités Sectoriales Locales (COSEL). De su constitución y funciones. La Gaceta diario oficial del gobierno de la república de Costa Rica, $N^{\circ} 146$ del 29 de julio del 2005. Recuperado https://www.imprentanacional.go.cr/ pub/2005/07/29/comp_29_07_2005.pdf

Instituto Nacional de Estadística y Censos. (2016). ENAHO. 2016. Indicadores de pobreza multidimensional según zona y región de planificación, julio 2016. Instituto Nacional de Estadística y Censos. C.R. Recuperado de http://inec.cr/encuestas/encuesta-nacional-de-hogares.

Instituto Nacional de Estadística y Censos. (2015). ENAHO. 2015. Distribución de los hogares por zona y región de planificación según condición de pobreza multidimensional. Instituto Nacional de Estadística y Censos. C.R. Recuperado de http://www.inec.go.cr/ encuestas/encuesta-nacional-de-hogares

InstitutoNacionaldeEstadísticayCensos.(2014).CensoNacionalAgropecuario: Fincas y personas productoras personas productoras por nivel de instrucción, según provincia y cantón. Recuperado de http://inec.cr/ agropecuario?title $=\&$ shs_term_node_tid_depth $=$ All\&field periodo tid $=$ All\&field_anio_documento_value $\overline{0} \% 5 \bar{B}$ value $\% 5 \mathrm{D} \% 5 \mathrm{Bdate} \% 5 \mathrm{D}=$

Instituto Nacional de Estadística y Censos. (2016). ENAHO. 2016. Asistencia a educación regular y nivel educativo de la población según zona y región de planificación, julio 2016. Recuperado de http:/www.inec.go.cr/educacion

Marín-Raventós, N. (2012, 21 de octubre). Educación y competitividad. La Nación. Recuperado de http://www.nacion.com/opinion/foros/ Educacion-competitividad_0_1300470036.html

Ministerio de Planificación Nacional y Política Económica. (2014). Plan Nacional de Desarrollo 2015-2018 “Alberto Cañas Escalante”. Ministerio de Planificación Nacional y política Económica, San José, Costa Rica. Recuperado en https://documentos.mideplan. go.cr/alfresco/d/d/workspace/SpacesStore/cd1 da 1b4-868b4f6f-bdf8-b2dee0525b76/PND\%202015-2018\%20Alberto\%20 Ca\%C3\%B1as\%20Escalante\%20WEB.pdf

Proyecto "Fortalecimiento de capacidades locales para la gestión de proyectos en organizaciones sociales del sector agropecuario en Guanacaste". (2015). Diagnóstico de Necesidades de Capacitación 
ReVista Universidad en Diálogo • Vol. 7, N. ², Julio-Diciembre, 2017, pp. 73-96

ISSN 2215-2849 • EISSN: 2215-4752

DOI: http://dx.doi.org/10.15359/udre.7-2.4

en TIC's. [Documento ubicado en archivos del Drive del Proyecto Fortalecimiento de Capacidades Locales para la gestión de proyectos en organizaciones sociales del sector agropecuario en Guanacaste, Sede Regional Chorotega, Universidad Nacional de Costa Rica].

Secretaría Ejecutiva de Planificación Sectorial Agropecuaria [SEPSA]. (2011). Política de estado para el sector agroalimentario y el desarrollo rural costarricense 2010-2021. SEPSA-MAG. San José, Costa Rica. Recuperado en http://www.mag.go.cr/bibliotecavirtual/a00289.pdf

Secretaría Ejecutiva de Planificación Sectorial Agropecuaria y Área de Política Agropecuaria y Rural. (2015). Plan Sectorial de Desarrollo Agropecuario y Rural 2015-2018. Recuperado en http://www.mag. go.cr/bibliotecavirtual/E14-10830.pdf

Suñol, S. (2006). Aspectos teóricos de la competitividad. Ciencia y sociedad, 32(2), 179-198. Recuperado de: http://www.redalyc.org/ articulo.oa? $\mathrm{id}=87031202$

Tam, N. (2016, 6 de febrero). La educación como pilar para la competitividad. La Estrella de Panamá. Recuperado de http://aestrella.com.pa/ economia/educacion-como-pilar-para-competitividad/23920368

Urquijo, M. (2014, junio). La teoría de las capacidades en Amartya Sen. EDETANIA, 46, 63-80. Recuperado de https://dialnet.unirioja.es/ servlet/articulo? codigo $=5010857$

Zúñiga, S. y Lezcano, S. (2015). Región Chorotega: Proyectos y espacios de articulación dirigidos a incidir en la generación de empleo, ingresos, acceso y calidad del agua para consumo humano. Guanacaste, Costa Rica. Universidad Nacional de Costa Rica. 